Instructions for authors, subscriptions and further details:

http://qre.hipatiapress.com

\title{
Aprendizaje de una Lengua Extranjera en Educación Obligatoria: Experiencias de Estudiantes con Sordera e Hipoacusia
}

Inmaculada Garrote Camarena ${ }^{1}$, Ricardo Moreno-Rodríguez ${ }^{1}$ \& Francisco Reyes Téllez ${ }^{1}$

1) Faculty of Social and Legal Sciences, Rey Juan Carlos University, Madrid.

Date of publication: February $28^{\text {th }}, 2021$

Edition period: February 2021 - June 2021

To cite this article: Garrote Camarena, l., Moreno-Rodríguez, R., \& Reyes Téllez, F. (2021). Aprendizaje de una Lengua Extranjera en Educación Obligatoria: Experiencias de Estudiantes con Sordera e Hipoacusia. Qualitative Research in Education, 10(1), 88-114. doi:10.17583/qre.2021.6641

To link this article: http://dx.doi.org/10.17583/qre.2021.6641

\section{PLEASE SCROLL DOWN FOR ARTICLE}

The terms and conditions of use are related to the Open Journal System and to Creative Commons Attribution License (CC-BY). 


\section{Learning a Foreign Language in Compulsory Education: Deaf and Hard-of-Hearing Students' Experiences}

Inmaculada Garrote Camarena Rey Juan Carlos University

Francisco Reyes Téllez

Rey Juan Carlos University
Ricardo Moreno-Rodríguez

Rey Juan Carlos University

(Received: 15 September 2020; Accepted: 23 February 2021; Published: 28 February 2021)

\section{Abstract}

The Spanish educational system is characterized by an increased emphasis placed on language teaching, apart from the majority language and other co-official languages. Furthermore, the educational reality is distinguished by the existing diversity within the classroom, increasing the complexity of learning a second language. This study analyses the opinions, beliefs and attitudes of deaf and hard-of-hearing students at university regarding their teaching-learning process of the English language during compulsory education. The data were collected through in-depth interviews, having as main topics the importance of learning the English language, as well as the experiences reported about didactic aspects in which they were involved. The results highlighted the basic conditions that must be met when deaf and hard-of-hearing students are learning a foreign language, in order to guarantee quality education promoting academic success.

Keywords: hearing loss, oral bilingualism, English language, compulsory education 


\section{Aprendizaje de una Lengua Extranjera en Educación Obligatoria: Experiencias de Estudiantes con Sordera e Hipoacusia}

Inmaculada Garrote Camarena Rey Juan Carlos University

Francisco Reyes Téllez

Rey Juan Carlos University
Ricardo Moreno-Rodríguez

Rey Juan Carlos University

(Recibido: 15 septiembre de 2020; Aceptado: 23 de febrero de 2021; Publicado: 28 de febrero de 2021)

\section{Resumen}

Uno de los aspectos que caracteriza el sistema educativo español es la creciente importancia que se le otorga a la enseñanza de otros idiomas, aparte de la lengua mayoritaria y otras lenguas cooficiales. Por otro lado, la realidad educativa actual se singulariza por la diversidad existente en el aula, incrementando la complejidad que ya conlleva el aprendizaje de una segunda lengua. El presente estudio analiza las opiniones, creencias y actitudes de estudiantes universitarios sordos y con hipoacusia en torno al proceso de enseñanza-aprendizaje de la lengua inglesa durante las etapas de enseñanza obligatoria, a través de la realización de entrevistas en profundidad, en las que se abordaba la relevancia del aprendizaje de la lengua inglesa y las principales experiencias relatadas sobre los aspectos didácticos de los que fueron participes. Los resultados exhibieron las condiciones básicas que deben concurrir durante el aprendizaje de la lengua inglesa en el que se cuente con alumnado con pérdida auditiva, con el fin de ofrecer una educación de calidad que favorezca el éxito académico.

Palabras clave: pérdida auditiva, bilingüismo oral, lengua inglesa, educación obligatoria. 


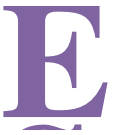

1 aprendizaje de idiomas extranjeros, entre los que se encuentra la lengua inglesa, alcanza su máxima representación con la Ley Orgánica 8/2013, de 9 de diciembre, para la mejora de la calidad educativa, así como en las leyes que se desprenden de ella, estableciéndose como uno de los ejes principales, a través del cual, se puede alcanzar la tan ansiada mejora educativa (Izquierdo, 2017). Precisamente, cumplir con los criterios que se establecen con respecto al aprendizaje de la lengua inglesa dentro del ámbito educativo, supone todo un reto, en el que se acentúa la complejidad cuando en el aula se encuentra alumnado con pérdida auditiva (Acosta, 2003; Bellés, 1995; Díaz-Estebánez \& Valmaseda, 1995; Domínguez, 2009; Fundación ONCE, 2018; Gómez, 2018; Martín-Pastor \& Durán-Martínez, 2019).

Cuando se aborda el tema de la sordera, no puede eludirse la concepción actual sobre la discapacidad en general y su influencia en las personas sordas, donde el énfasis no se ubica únicamente en los factores internos o personales, sino en las condiciones externas o ambientales que pueden ser generadoras de posibles limitaciones en cuanto a la participación del individuo. Esta idea es extensible al contexto educativo, donde debe ser éste el que ofrezca una respuesta acorde para que se lleve a efecto la igualdad de oportunidades y la participación igualitaria, tal y como se desprende de los principios de la inclusión educativa y atención a la diversidad. Además, la enorme heterogeneidad que se presenta entre las personas sordas y con hipoacusia debe tomarse en cuenta, dado que esto entraña la necesidad de ofrecer una respuesta lo más individualizada posible, que tome en consideración una gran variabilidad de realidades y dimensiones, provocando que una respuesta y marco de actuación generalizado no se ajusten a la totalidad del alumnado con pérdida auditiva (Alonso et al., 2009; Díaz-Estébanez \& Valmaseda, 1995).

\section{Necesidades Educativas de las Personas con Sordera e Hipoacusia}

El aprendizaje de la lengua inglesa, en el caso de las personas sordas, es un requerimiento y, por ende, una necesidad de cara a su inclusión plena en el sistema educativo, encontrándose estrechamente relacionado con el éxito académico, que dará lugar a su posterior decisión a la hora de dar continuidad a estudios superiores, aspecto que no tiene que ser excluyente con respecto al desarrollo y utilización de su lengua natural (Domínguez \& 
Alonso, 2004). Lo que cabe determinar es el modo en el que esto se está llevando a cabo, teniendo en cuenta los aspectos que los estudiantes con pérdida auditiva requieren, para que se efectúe un aprendizaje significativo y ajustado a sus necesidades. El aprendizaje de la lengua oral en este tipo de alumnado ha estado principalmente caracterizado por el enfoque audiológico, en el que se ha puesto el foco de la actuación en el aprovechamiento de los restos auditivos, con los ajustes tecnológicos disponibles, el uso de sistemas alternativos y aumentativos de la comunicación oral (Domínguez \& Alonso, 2004), al mismo tiempo que se aplicaban métodos de enseñanza prácticamente similares a los de los alumnos oyentes, ocasionando unos índices de fracaso escolar considerables (Herrera, 2014). Actualmente, para que se produzca el acceso a la lengua oral, ya sea una L1 (en este caso el castellano como primera lengua) o L2 (segunda lengua oral), deben coexistir unas condiciones, recursos y apoyos específicos (Acosta, 2001).

Algunos de los obstáculos contextualizados en las aulas en las que se imparte la materia de inglés como primera lengua extranjera están vinculados con el acceso a la información y a la comunicación, principalmente, debido a la utilización de la lengua oral, el peso que se le otorga como vehículo de transmisión de conocimientos e información y como código comunicativo para establecer interacciones con sus compañeros y profesores. A partir de esto, se debe acudir a los diversos sistemas para garantizar el acceso a la comunicación e información (DíazEstébanez \& Valmaseda, 1995; Kontra et al., 2015), en el que deben contemplarse otros criterios didácticos y metodológicos, de necesaria aplicación durante la impartición de la asignatura de inglés, cuando se encuentre entre el alumnado estudiantes sordos y/o con hipoacusia. Domínguez y Alonso (2004) proponen como ejemplos de estos criterios los que se detallan a continuación: los principios que rijan la presencia de la Lengua de Signos Española (LSE) en la escuela y que se muestre el mismo estatus con respecto a las lenguas orales; asegurar la disponibilidad de los recursos tanto materiales como profesionales que son necesarios en la atención educativa para las personas sordas y la comprobación sistemática a la hora de garantizar una accesibilidad a la comunicación e información real en todos los ámbitos, lugares y momentos del centro educativo, entre otros.

Si bien la lengua inglesa es el objeto de estudio, también tiene que valorarse la lengua que se va a utilizar como medio de transmisión de 
conocimientos. Habitualmente, es la propia lengua inglesa la que se utiliza como medio de emisión de información y establecimiento de interacciones comunicativas que surgen en el aula, ya que permite ofrecer al alumnado contextos más variados y ricos, además de una inmersión real (Domínguez \& Alonso, 2004; Izquierdo, 2017; Laorden \& Peñafiel, 2010); pero en el caso de las personas sordas, existen varias posibilidades, cuya conveniencia es relevante analizar. Una de estas opciones es la reflexión sobre la incorporación de la Lengua de Signos Española en el aula de inglés, tal y como se está llevando a cabo en algunos centros de educación bilingüe (LSE-lengua castellana), y la otra opción, es la utilización simultánea de las dos lenguas orales, la inglesa y la castellana, siendo necesaria una profunda reflexión sobre su idoneidad por parte de los profesionales implicados, así como el conocimiento de nivel competencial de los estudiantes sordos en lengua castellana (Domínguez \& Alonso, 2004; Kontra et al., 2015; Quay, 2005).

Ya se ha puesto de manifiesto la relevancia que supone el aprendizaje de una lengua extranjera oral, como es el inglés, también para las personas sordas y con hipoacusia (Berent, 2001). Asimismo, son muchos los aspectos que deben contemplarse y de muy diversa índole, desde las características personales, culturales, lingüísticas, del entorno familiar, educativo y social, y las necesidades educativas que se presentan a la hora de aprender un idioma oral. A pesar de todo lo expuesto, son escasas las referencias concretas que faciliten y muestren pautas específicas que los docentes encargados de la enseñanza del inglés apliquen para garantizar la igualdad de oportunidades, e incluso observándose en algunos momentos, ciertas incoherencias que dificultan una práctica inclusiva. Asimismo, se otorga en la legislación educativa un especial hincapié a la función comunicativa $\mathrm{y}$, por tanto, al aspecto oral de las lenguas, dentro del aprendizaje que tiene como objeto de estudio la lengua inglesa, subsanándose en la actualidad con la exención de las competencias lingüísticas que entrañan dicha funcionalidad comunicativa y con la recepción/comprensión y producción de mensajes orales.

\section{Metodología}

Tras el planteamiento del principal propósito de esta investigación, es decir, inspeccionar y valorar aspectos educativos significativos vinculados con las 
necesidades que requieren los estudiantes con pérdida auditiva, al mismo tiempo que se profundiza en el conocimiento de sus experiencias educativas, durante el proceso de enseñanza-aprendizaje de un segundo idioma oral en la escuela, se determinó que el método que resultaba más adecuado fuera la investigación cualitativa. Precisamente esta elección se debe a su capacidad para indagar y ahondar en ciertos ámbitos, entre los cuales también se encuentra el educativo y las problemáticas que se hallan en él, lo cual colabora a interpretar la realidad y comprender las dimensiones que configuran el tema objeto de estudio (Lankshear \& Knobel, 2000; Munarriz, 1992). Además, a través del método cualitativo se ofrece la posibilidad de analizar los puntos de vista de los sujetos de estudio en profundidad y establecer conexiones que se utilizan para la realización de interpretaciones e inferencias, así como explicaciones y predicciones (Lankshear \& Knobel, 2000; Munarriz, 1992).

\section{Muestra}

Para la selección de la muestra se utilizó el muestreo no probabilístico estratégico, ya que las unidades muestrales atienden a criterios relacionados con los objetivos planteados en el estudio (Pérez-Llantada \& López de la Llave, 2001), también referido por Maxwell (2012) como purposeful sampling, por lo que lo relevante no es la cantidad de sujetos participantes, sino la información que cada uno de ellos puede aportar a esta investigación, ya que son parte esencial de la realidad que se pretende comprender. Así pues, la población objeto de estudio son alumnos y alumnas universitarios con sordera o hipoacusia matriculados durante el periodo de realización del presente estudio, comprendiendo desde el curso académico 2016-2017 hasta el 2019-2020 y que estuvieran realizando estudios de Grado o Máster en la Universidad Rey Juan Carlos. Asimismo, la muestra tenía que haber formado parte de la Unidad de Atención a Personas con Discapacidad y Necesidades Educativas Especiales (NEE) de esta misma institución, dado que son los estudiantes que forman parte de dicho servicio aquellos que, o bien previo a la prueba de acceso a la Universidad o durante la realización de sus estudios superiores, han solicitado la necesidad de ciertos apoyos, para poder garantizar su seguimiento del ritmo de aprendizaje dentro del aula. A estos criterios de inclusión se añaden los siguientes: 
1. Sujetos que tengan diagnosticada únicamente hipoacusia o sordera, con un grado de discapacidad asociado igual o superior al $33 \%$.

2. Estudiantes que hayan cursado la asignatura curricular de primera lengua extranjera: inglés en la etapa de educación obligatoria (Primaria y/o Secundaria).

3. Estudiantes cuya aparición de la pérdida auditiva haya tenido lugar en edades tempranas, previas o durante su escolarización en la etapa de educación obligatoria.

Finalmente, la muestra de este estudio se compuso de un total de 15 participantes, de los cuales un $66,7 \%$ de la totalidad fueron mujeres, mientras que el restante 33,3\% eran hombres. La media de edad fue de 27,4 años, situándose todos en una horquilla de edades muy amplias, comprendiendo tres décadas. Con respecto a la lengua materna, se contemplaron tres opciones, obteniendo un 53,3\% del total de los participantes que son usuarios exclusivamente de la Lengua de Signos Española (LSE), un 33,3\% utilizan la lengua oral castellana y un $13,4 \%$ se catalogaron como bilingües, dado que hacen uso de manera indistinta tanto de la lengua oral castellana como de la LSE. Los datos referidos al grado de pérdida auditiva mostraron que la muestra se organizaba en torno a un $66,6 \%$ de los sujetos de la investigación que presentan sordera, un 26,7\% con hipoacusia severa y un $6,7 \%$ con hipoacusia moderada, a lo que debe añadirse un $73,3 \%$ de los mismos en los que la pérdida auditiva es prelocutiva, mientras que el $26,7 \%$ restante manifestó la hipoacusia posterior a la adquisición y desarrollo del lenguaje oral. La totalidad de ellos disponen de certificado de discapacidad, aunque únicamente un $40 \%$ de los estudiantes participantes entraron por el cupo de discapacidad a la universidad. Las ramas de conocimiento en las que se enmarcan las titulaciones universitarias que han cursado o se encontraban cursando los participantes fue mayoritariamente un $60 \%$ perteneciente a Ciencias Sociales y Jurídicas, un $20 \%$ en Ciencias de la Salud y un $6,7 \%$ en Ingeniería y Arquitectura, mientras que un 13,3\% estaba realizando titulaciones de Postgrado, más concretamente Másteres Oficiales (tabla 1). 
Tabla 1.

Distribución sociodemográfica de la muestra participante.

\begin{tabular}{|c|c|c|c|c|}
\hline $\begin{array}{c}\text { Código } \\
\text { Participante }\end{array}$ & & & bles sociodemográficas & \\
\hline & Sexo & Edad & Lengua materna & $\begin{array}{l}\text { Momento de } \\
\text { aparición }\end{array}$ \\
\hline 001 & $\mathrm{M}$ & 20 & Lengua oral castellana & 5 años \\
\hline 002 & M & 25 & Bilingüe & 2 años \\
\hline 003 & $\mathrm{H}$ & 21 & LSE & nacimiento \\
\hline 004 & M & 29 & Lengua oral castellana & 9 años \\
\hline 005 & $\mathrm{H}$ & 30 & Lengua oral castellana & 7 años \\
\hline 006 & M & 23 & LSE & nacimiento \\
\hline 007 & M & 35 & LSE & nacimiento \\
\hline 008 & $\mathrm{H}$ & 28 & Bilingüe & nacimiento \\
\hline 009 & M & 28 & LSE & nacimiento \\
\hline 010 & M & 20 & LSE & nacimiento \\
\hline 011 & M & 21 & Lengua oral castellana & 1 año \\
\hline 012 & M & 20 & Lengua oral castellana & 6 años \\
\hline 013 & $\mathrm{H}$ & 46 & LSE & nacimiento \\
\hline 014 & $\mathrm{H}$ & 35 & LSE & nacimiento \\
\hline 015 & M & 30 & LSE & nacimiento \\
\hline
\end{tabular}




\section{Técnicas y Procedimiento}

Los motivos por los cuales se ha elegido como instrumento la entrevista en profundidad para recopilar información atienden a que puede tratarse de un modo provechoso de acceso a opiniones, creencias y experiencias de los participantes en el ámbito de la educación, además de la obtención de datos significativos para el estudio, debido a cierta flexibilidad que permite abordar temas y profundizar en aspectos que previamente no se habían contemplado (Lankshear \& Knobel, 2000; Munarriz, 1992). Adicionalmente, tal y como establecen Taylor y Bodgan (1987), la entrevista permite estudiar acontecimientos del pasado y disponer de un mayor aprovechamiento del tiempo. Para cometer tal fin se preparó el contexto en el que va a tener lugar la entrevista por parte del investigador (Taylor \& Bogdan, 1987), contando con un guión previo con un listado de preguntas. Ambos participantes, investigador y entrevistado, mantienen una interacción comunicativa con la utilización de sus propias palabras y expresiones, en términos similares en los que acontece una conversación habitual. Esto atiende a la necesidad de tratar los temas que se estimaban fundamentales para el desarrollo de la investigación, lo cual ofrece al entrevistado la posibilidad de expresarse y comentar todo aquello que estime necesario incluir, explicar, reflexionar o desarrollar (Munarriz, 1992) que, en este caso, configura parte de su experiencia educativa y su relación en dicha etapa con la lengua inglesa.

Para el cumplimiento de los objetivos se elaboró un guión previo a la realización de la propia entrevista que recoge un total de 34 cuestiones, que varían dependiendo del desarrollo de la entrevista de cada participante. Para la elección de las preguntas definitivas se llevó a cabo una revisión por parte de cuatro expertos ampliamente relacionados con el ámbito de estudio. Dos intérpretes en Lengua de Signos Española, uno de ellos Doctor y docente del Grado de LSE y Comunidad Sorda. El otro experto forma parte de la denominada Cultura Sorda por ser hijo de padres sordos; a esto debe añadirse un Doctor en Terapia Ocupacional y el Técnico de la Unidad de Atención a las personas con Discapacidad de la Universidad Rey Juan Carlos, profesional clave en la solicitud y gestión de las adaptaciones que se aplican en el examen de Idioma Moderno. Todos ellos revisaron las preguntas propuestas en términos de conveniencia y relación con el tema de la investigación, obteniendo ligeras modificaciones de redacción y 
planteamiento de las cuestiones, para mejorar la comprensión por parte del entrevistado. Con el fin de corroborar este proceso se realizó una entrevista piloto a dos estudiantes que finalmente no contaron como muestra.

Las preguntas eran de dos tipos principales, descriptivas, con el propósito de que los participantes puedan comentar lo que se plantea en las cuestiones, sin ningún tipo de condicionamientos. Por otro lado, preguntas que comparten características con los denominados "relatos solicitados", que según Taylor y Bogdan (1987) proveen descripciones de acontecimientos y experiencias personales pasadas de la infancia y la adolescencia. Esta guía supuso el inicio del proceso de categorización, estableciendo una serie de categorías previas a la realización de las entrevistas y que dan lugar a los principales bloques temáticos (Maxwell, 2012).

El primer tema comprende un total de once preguntas base, las cuales abordan diversos aspectos sobre la concepción y opiniones que tienen los entrevistados acerca del aprendizaje de una segunda lengua en términos globales, y de la lengua inglesa en particular. El segundo bloque temático disponía de un total de dieciocho preguntas que versaban sobre el aprendizaje del inglés durante la etapa de escolarización obligatoria Primaria y Secundaria-, en la que se solicitó información de relevancia sobre aspectos metodológicos, didácticos y emocionales vinculados con la asignatura de inglés, sus profesores y resto de compañeros. El tercer y último bloque del guión era muy breve, presentando un total de cinco preguntas, que persiguieron mostrar las principales propuestas de mejora, además de reflexiones que los propios sujetos de la investigación proponen.

Una vez planteado el estudio, se realizó un registro de todos aquellos alumnos y alumnas con sordera e hipoacusia que cumplían con los criterios de inclusión. Al mismo tiempo se procedió a la localización de los espacios en los que iban a tener lugar las entrevistas. Las principales características de las salas eran que cuentan con mobiliario ergonómico y condiciones óptimas de iluminación, además de brindar la posibilidad de establecer conversaciones privadas sin interrupciones ni elementos distractores que facilitasen que el participante se sintiera relajado y cómodo para poder expresarse con total libertad (Taylor \& Bogdan, 1987).

El periodo en el que se han realizado las entrevistas ha sido desde el 15 de septiembre del 2018 hasta el 31 de octubre de 2019. De acuerdo con la extensión de las entrevistas, aun con duraciones variables en cada una de 
ellas, ha sido desde una hora hasta una hora y cincuenta minutos. El procedimiento de recopilación, transcripción y análisis propio de la investigación cualitativa requiere de gran cantidad de tiempo, a la vez que se genera un gran número de información (Fernández Núñez, 2006). De hecho, el periodo total en el que se han llevado a cabo las transcripciones de las entrevistas ha comprendido desde el 17 de septiembre de 2018 hasta el 5 de noviembre de 2019, ya que el proceso de transcripción se efectuaba de manera simultánea a la realización de cada una de las entrevistas. Posteriormente, la información recabada fue codificada en subcategorías en concordancia con los temas principales anteriormente detallados, con el fin de proceder al análisis de las mismas y que se incluyen en la tabla 2 . Durante el proceso de análisis-categorización-interpretación se ha mantenido vigente los posibles sesgos que el investigador cualitativo puede acometer, distanciándose de todo aquello que no surja o forme parte de la denominada 'descripción protocolar' (Martínez Miguélez, 2006). 
Tabla 2.

Categorías y subcategorías de la entrevista en profundidad.

\section{Categorías}

Subcategorías

Relevancia de su aprendizaje.

Interés por su aprendizaje.

Concepción y opiniones sobre el aprendizaje de: lenguas extranjeras orales y de signos.

Aspectos metodológicos, didácticos y emocionales vinculados con el aprendizaje del inglés durante Primaria y Secundaria.

Propuestas de mejora y reflexiones finales.
Beneficios obtenidos del conocimiento de una o más lenguas.

Oportunidades laborales asociados a la competencia lingüística de la lengua inglesa.

Tipología de centro educativo.

Presencia de la LSE.

Ubicación y disposición del aula.

Adaptaciones de acceso y curriculares.

Percepción sobre el grado de inclusión.

Interacción comunicativa entre alumno-profesor.

Mejoras metodológicas.

Requisitos esenciales para involucrase en su estudio.

Percepción sobre la lengua inglesa. 


\section{Resultados y Discusión}

\section{Importancia y Motivación por Aprender la Lengua Inglesa}

Los participantes entrevistados afirmaron en su gran mayoría la importancia de aprender un segundo idioma, independientemente de las características que tenga la L2. Esto refleja que las personas con pérdida auditiva participantes de esta investigación son plenamente conscientes de la creciente relevancia que se ha ido otorgando al aprendizaje de uno o varios idiomas y que se ha ido extendiendo a lo largo del territorio español, impulsado también por Europa (De Castro, 2014; Fernández Agüero, 2009; Izquierdo, 2017; Lova et al., 2013). Esto se encuentra reforzado por los resultados de las entrevistas en las que se corrobora el interés de los estudiantes participantes por aprender una lengua extranjera oral, encontrándose muchos de ellos realizando estudios paralelos, o habiéndolos finalizado recientemente, debido a la asignatura de Idioma Moderno que deben cursar en sus estudios universitarios.

\footnotetext{
Sí, sí. Sí, está claro que sí, porque, bueno, yo veo que tengo capacidad, tengo esa capacidad y a mí me gustaría también, y que reconocieran también que tenemos esa capacidad de aprender otros idiomas (extracto entrevista sujeto 006).
}

Los estudiantes con sordera e hipoacusia también pusieron de manifiesto una serie de motivos y beneficios que avalan la relevancia de aprender un idioma extranjero, en general, y en particular el caso del inglés, en el que se incluye la función comunicativa con un número mayor de hablantes, que viene a sostener la necesidad de establecer interacciones comunicativas por parte de los individuos, no sólo con el entorno más cercano, sino también con personas pertenecientes a otros territorios y culturas (Izquierdo, 2017); la resolución de situaciones en el extranjero, el enriquecimiento cultural y personal que entraña, también contemplado en los diversos esfuerzos y normativa establecida en pro del bilingüismo; además del acceso a la información, destacable dado el perfil al que se está sometiendo a análisis.

Hace poco me he ido a Nueva York, o sea, sí que es verdad que hay gente que habla español, pero hay como una barrera, entonces si no 
hablas inglés, es como que yo que sé, que te sientes como cohibido a la hora de pedir, yo que sé, un café, ya te sientes un poco cohibido porque como no sabes decirlo o lo que sea; pues eso, un poco hay un muro y una especie de barrera (extracto entrevista sujeto 005).

Otro aspecto son las oportunidades laborales que ofrece el contar con conocimientos en una lengua que no sea la materna, en el que los alumnos vincularon el aprendizaje de la lengua inglesa con el incremento de posibilidades laborales cuando se aludía tanto a las personas en general como a sus casos en particular, ya que no se puede eludir su significancia como una de las características más representativas de la sociedad actual, con unas fronteras cada vez más difusas, en las que el conocimiento de otros idiomas, a parte del materno, posibilita mayores oportunidades laborales (Izquierdo, 2017; Julián-de-Vega \& Ávila-López, 2018).

\section{El Contexto Educativo como Eje Prioritario en el Aprendizaje de una}

\section{L2}

En primer lugar, se han observado los diferentes entornos educativos en los que los estudiantes participantes de la investigación han desarrollado el aprendizaje de la lengua oral inglesa, durante la etapa de educación obligatoria. Es precisamente aquí donde cobra una especial relevancia el contexto en el que va a tomar lugar el proceso de enseñanza-aprendizaje, cuyo principal propósito es desarrollar y alcanzar el máximo progreso de todos los alumnos, siendo por ello necesario que dicho espacio cumpla con los requisitos que ya se estipulan desde la Convención sobre los derechos de las Personas con Discapacidad (ONU, 2006), que contribuye en gran medida a la hora de considerar a las personas con discapacidad como sujetos de derechos que deben poder disfrutar y ejercer, a través de una participación plena, como miembros activos de la sociedad, en el que se incluye sin lugar a dudas, la educación (Domínguez, 2016; Echeita \& Verdugo, 2004; Kontra et al., 2015; Palacios \& Bariffi, 2007; RodríguezOrtiz, 2005; Sanjosé Gil, 2007; Verdugo, 2011).

Por tanto, la mayoría han acudido a los denominados centros de escolarización preferente a alumnado sordo y con hipoacusia. Una parte significativa de los estudiantes sordos acudieron a una categoría específica 
que se enmarca dentro de este tipo de escolarización preferente, es decir, los denominados de enfoque bilingüe-educación compartida, lo cual pone de manifiesto la importancia del contexto educativo de las personas sordas, donde este tipo de centros, cumplen con la inclusión de otros pares oyentes y cuentan con una larga experiencia en la atención a este tipo de estudiantes, pudiendo ofrecer una educación que se ajuste a sus necesidades (Díaz-Estébanez \& Valmaseda, 1995; Domínguez, 2009). Otras modalidades que se presentaron fueron colegios ordinarios y centros de educación especial específicos para personas sordas, aunque la totalidad de estos últimos estudiantes ratificaron que dichos centros específicos se han transformado en colegios de escolarización preferente a alumnado con pérdida auditiva o bien cerraron sus puertas, lo que viene a reafirmar la modificación estructural del sistema educativo hacia centros educativos más inclusivos. De todas formas, para que se pueda hablar del cumplimiento de los principios de igualdad de oportunidades, participación plena y mejora de la calidad, el sistema educativo ordinario debe estar preparado para atender la diversidad presente en las aulas, evitando cualquier tipo de segregación y la eliminación de cualquier tipo de barrera que se identifique en el contexto escolar, en el que se ofrezca un amplio abanico de posibilidades que, al fin y al cabo, van a suponer oportunidades de aprendizaje para todo los estudiantes (Blanco \& Duk, 2011; Deaño, 2004; Garzón et al., 2016; Giné, 2004; Parrilla Latas, 2004; Soriano, 2011).

Vinculado a este hecho se encuentra la presencia de otros compañeros con pérdida auditiva, donde la totalidad de participantes que acudieron o bien a centros preferentes o colegios especiales específicos para personas sordas, contaban con más compañeros con las mismas características auditivas; mientras que aquellos que acudieron a colegios ordinarios sin ningún tipo de especificidad, se evidencia el hecho de que la totalidad de la muestra no tuvo más compañeros con sordera e hipoacusia en el mismo aula, donde únicamente dos estudiantes mencionaron que en el colegio había algún alumno con pérdida auditiva. Esto muestra una tendencia en la que, aun pudiendo optar a cualquier centro ordinario, cuyos principios educativos a nivel nacional están obligados a atender la diversidad y contemplar cualquier tipo de necesidad educativa, la mayoría de estos estudiantes han optado por los centros denominados de escolarización preferente. Asimismo, se debe ampliar dicha responsabilidad a la hora de abordar la diversidad lingüística y cultural, que se exhibe en la importancia 
que se otorga al aprendizaje de idiomas extranjeros (Julián-de-Vega \& Ávila-López, 2018) pero en el que se lleve a cabo los principios inclusivos y un aprendizaje significativo de la lengua inglesa (Bellés, 1995; Domínguez, 2009; Fundación ONCE, 2018; Martín-Pastor \& DuránMartínez, 2019).

\section{Principales Cuestiones Metodológicas y Prácticas de los Docentes de Inglés}

De la misma manera resulta necesario conocer las percepciones que mostraron los estudiantes sobre la suficiencia de las horas de inglés que han cursado e impartido. Gran parte de los alumnos universitarios entrevistados dijeron que las horas que tuvieron no eran suficientes, aportando como principales motivos el hecho de que deberían incrementarse, desde su perspectiva actual, al mismo nivel de otras materias curriculares que tenían mayor peso, como el caso de las matemáticas y lengua y literatura, argumentando que un idioma no se puede consolidar, ni asimilar correctamente con las horas establecidas.

Creo que de hecho tendría que ser mucho más, mucho más en plan de tres o toda o todos los días, igual que matemáticas y lengua, pues igual el inglés. Y más que nada porque como ahora ya se pide inglés, pues creemos una base de inglés (extracto entrevista sujeto 004).

Otro criterio metodológico significativo es la ubicación de los estudiantes con pérdida auditiva en el aula, reflejado también en las respuestas de los estudiantes participantes, aportando como distribuciones más habituales la organización en filas. Los estudiantes que mostraron preferencia por esta estructura mencionaron su predilección por ubicarse en las primeras filas, debido a que se facilitaba la visibilidad de los espacios más significativos, como es el caso del profesor y la pizarra, permitiendo la lectura labiofacial o el posicionamiento del intérprete (Alonso et al., 2009; Domínguez \& Alonso, 2004; Herrera, 2014). 
También, me facilita mucho la lectura labial de algunos profesores, si hay alguna cosa que no... pues lectura labial. Y yo creo que, por eso, porque me gusta verlo todo (extracto entrevista sujeto 011 ).

Otros comentaron la organización de los pupitres en forma semicircular, la totalidad de ellos mostrando los beneficios y conformidad con dicha distribución.

Entonces claro, nos veíamos los compañeros y también qué decía el profesor, a los alumnos, entonces eso sí que estaba muy bien (extracto entrevista sujeto 007).

Durante la etapa de Primaria, los estudiantes entrevistados reiteraron que realizaban las mismas actividades que el resto de sus compañeros, aunque debe mencionarse aquellos que estaban exentos del listening y que realizaban otras tareas alternativas.

Claro, yo evidentemente no hacía el listening, entonces me ponían una lectura, como una doble lectura, me compensaban con una doble lectura, mientras los compañeros hacían el listening (extracto entrevista sujeto 010).

En otros casos, se procedía al agrupamiento de los alumnos con pérdida auditiva para que, durante una hora de la asignatura de inglés, acudiesen a clases de apoyo de otras materias.

Los oyentes tenían tres horas y nosotros dos horas, esa tercera era como una especie de apoyo particular que nos habían dado porque el profesor con el que estábamos no tenía ni idea de inglés; entonces eran apoyos para otras asignaturas (extracto entrevista sujeto 006)

En esta ocasión, la mayoría de los entrevistados afirmaron hacer las mismas tareas en la etapa de Secundaria, teniendo que tomar en cuenta aquellos estudiantes que estaban exentos de la realización de actividades donde se desarrollasen las competencias de comprensión auditiva.

Sí, era totalmente distinto, los deberes, por ejemplo, nos mandaba, por ejemplo, hacían un listening, todos los compañeros estaban 
escuchando, otros nos daban algo para ir leyendo, repitiendo siempre lo mismo, por ejemplo, no hacíamos trabajo de comprensión o de expresión, sino que leíamos (extracto entrevista sujeto 015).

Debe añadirse la alusión a la creciente dificultad observada por parte de los entrevistados con respecto al paso de Primaria a Secundaria.

El nivel subió en Secundaria, mucho más trabajo y dedicación (extracto entrevista sujeto 015).

Y a los efectos que esto tenía en sus emociones.

Y luego ya en Secundaria, pues sí que es verdad que, a lo mejor, pues eso, ya te empiezas a frustrar más, es más difícil (extracto entrevista sujeto 004).

En estrecha relación, se encuentran las principales adaptaciones que se llevan a cabo durante la impartición de esta materia, teniendo en cuenta, que dichas modificaciones sean asumibles por parte de los centros y que reflejen una atención personalizada y teniendo presentes las necesidades existentes, con un especial énfasis en los estudiantes con pérdida auditiva, sobre todo, en el aprendizaje de un idioma oral (Blanco \& Duk, 2011; Casanova, 2011; González-Gil, 2011; Fernández Agüero, 2009; Fundación ONCE, 2018). Los propios alumnos plantearon diez tipos de adaptaciones, obteniendo mayor representación la exención del listening, la presencia del intérprete en LSE, la adaptación de los materiales didácticos, ya fueran fotocopias con tareas alternativas o libros de texto diferentes al resto de estudiantes, la realización de exámenes vinculados al nivel de los materiales, la utilización del sistema de Frecuencia Modulada (FM) y la escritura en la pizarra de gran parte de la información aportada durante las clases. Se recibieron respuestas por parte de los alumnos participantes, cuya lengua materna es la lengua oral castellana, en el que se ratificó la dificultad que entrañaban para ellos las clases en las que se impartía en su totalidad en lengua inglesa, resultándoles más sencillo el seguimiento de la dinámica del aula, con aquellos profesores que hacían uso de la combinación de ambas lenguas orales, reflejo de lo estipulado en las leyes educativas, tanto la LOMCE, como el Real Decreto 126/2014, de 28 de febrero y el Real 
Decreto 1105/2014, de 26 de diciembre, por el que se establecen el currículo de Primaria y Secundaria correspondientemente, en el que como medidas de flexibilización y adaptaciones, se contempla la utilización de las lenguas oficiales para aquellos alumnos que puedan presentar algún tipo de dificultad a la hora de la producción de la lengua oral.

Resulta apropiado retomar el apartado en el que se solicitó a los estudiantes con sordera e hipoacusia comentar las actividades que recordaban que les gustaba realizar y aquellas que no; mientras que se aportaron una gran diversidad de las que sí les gustaba hacer, se observó que de aquellos estudiantes que no estaban exentos del listening, las tareas en las que tenían que desarrollar la comprensión auditiva eran, sin lugar a dudas, aquellas hacia las que mostraron una mayor animadversión, incluso en los participantes que estaban exentos de su evaluación, pero que realizaban este tipo de trabajo en la dinámica habitual del aula. Sin embargo, el replantearse aquellas competencias lingüísticas que se deben potenciar en este alumnado no es una cuestión baladí (Quay, 2005), ya que entre las propuestas de mejora que incluyeron los estudiantes sordos y usuarios de LSE, se encuentra el potenciar las habilidades vinculadas con la escritura y la lectura.

El tema del listening entiendo que haya que quitarlo y añadir más práctica de escritura, para que compense unas horas con las otras (extracto entrevista sujeto 003).

\section{Presencia de la Lengua de Signos Española dentro del Aula de Inglés}

Un aspecto que no puede eludirse es la presencia de la Lengua de Signos Española en el aula de inglés, aspecto establecido en la Convención sobre los Derechos de las personas con discapacidad de 2006, la Declaración de Salamanca (UNESCO, 1994) y la Ley 27/2007, de 23 de octubre, por la que se reconocen las lenguas de signos españolas, siempre que se esté analizando la situación de las personas sordas y con hipoacusia (Alonso et al., 2009; Casanova, 2011; Fernández-Viader \& Fuentes, 2004; Palacios \& Bariffi, 2007; Verdugo, 2011), en el que resulta necesario, sobre todo, comentar que prácticamente la totalidad de la muestra de estudiantes con pérdida auditiva son usuarios, se encuentran estudiando dicha lengua, o 
bien tienen conocimientos básicos; frente a un estudiante participante que no conoce la LSE.

A colación de lo establecido, conviene mencionar que aquellos alumnos que fueron a centros que no eran de escolarización preferente, mayoritariamente no tuvieron ningún tipo de contacto con esta lengua; aunque cabe matizar que la lengua materna de la totalidad de ellos es la lengua oral, a excepción de un participante, usuario de la LSE, quien determinó que contaba con un intérprete en la totalidad de las materias. Por el contrario, los alumnos que fueron a centros de escolarización preferente a alumnado con discapacidad auditiva con enfoque bilingüe, la totalidad de ellos mencionaron la presencia de esta lengua en las clases de inglés. La mayoría de los participantes usuarios de la Lengua de Signos Española mantuvieron la necesidad de que estos docentes hubiesen contado con más conocimientos en LSE.

En clase no entendíamos nada, porque el profesor no estaba muy capacitado en lengua de signos, era un nivel muy flojo de lengua de signos, entonces no explicaba con claridad, no sabía nada de lengua de signos, ni de Cultura Sorda, por ejemplo (extracto entrevista sujeto 014).

Un elemento que los alumnos con sordera e hipoacusia comentaron es la presencia del intérprete, pero resulta fundamental que quien ejerza dicho rol, tenga conocimientos de inglés, ya que algunos de los datos obtenidos evidenciaron experiencias negativas.

El profesor hablaba en inglés, claro entonces, el intérprete claro, el intérprete no sabía inglés, claro no se enteraba de nada, entonces, entonces el intérprete no sabía cómo hacer, no podía interpretar (extracto entrevista sujeto 006).

A pesar de la idoneidad que supone la presencia de la LSE en el aula de inglés, no puede eludirse el menor grado de exposición a un idioma extranjero por parte de los usuarios de LSE, por lo que el planteamiento de introducir alguna variante puede ser tomado con especial cuidado. Para ello, se solicitó a los estudiantes sordos y usuarios de la Lengua de Signos Española y bilingües su opinión acerca del aprendizaje de otras lenguas de signos o del Sistema Internacional de Signos (SSI), observándose que la 
totalidad de estos participantes realizaron unas valoraciones positivas, incluso proponiendo como mejora la presencia de un intérprete de SSI u otras lenguas de signos extranjeras. A esto debe añadirse el hecho de que los entrevistados sordos, cuya lengua única es la LSE o bilingües de ambas lenguas (LSE y lengua española) destacaron la conveniencia de la presencia del intérprete, pero que este disponga de conocimientos en lengua inglesa o en Sistema Internacional de Signos, lo que se configura como una propuesta de mejora altamente significativa.

Yo pienso que el intérprete en lengua de signos, eh...debería ser en internacional, pienso que sería lo mejor, lo idóneo (extracto entrevista sujeto 003).

\section{Conclusiones}

Con respecto a las opiniones, creencias y actitudes de los estudiantes universitarios, se determina que los alumnos con pérdida auditiva, participantes de este estudio, otorgan una gran relevancia al aprendizaje de la lengua inglesa, mostrando el papel significativo que éste entraña a la hora de obtener mayores oportunidades laborales, acceso a un número mayor de información, el conocimiento de otras culturas y la posibilidad de establecer interacciones comunicativas con individuos pertenecientes a otros países. Asimismo, mayoritariamente, han valorado su relación con los docentes de inglés de manera positiva, sin embargo, hay un incremento significativo de alumnos que aportan información negativa cuando evalúan su relación con la lengua inglesa.

Como principales experiencias y propuestas de mejora que plantean los sujetos de estudio entrevistados, destacan la eliminación de actividades en las que se desarrolla la competencia lingüística de comprensión auditiva, lo que lleva a pensar que esta práctica docente, sí entraña ciertas consecuencias negativas en el alumnado con sordera e hipoacusia. Por ello, se presenta como necesario la mejora de las condiciones acústicas y tecnológicas durante la realización de actividades cuyo principal propósito sea el desarrollo de la comprensión auditiva. Adicionalmente, es conveniente ubicar en el centro de las reflexiones docentes, las competencias lingüísticas que se tienen que potenciar con respecto al 
alumnado sordo, así como el refuerzo de las destrezas lingüísticas asociadas a la lectura y escritura.

También resulta necesario determinar la presencia de la LSE en el aula de inglés, en el que la totalidad de estudiantes sordos han recalcado la importancia de la utilización de la Lengua de Signos Española como recurso metodológico dentro del aula de inglés, ya sea por parte del intérprete o que el propio docente de inglés sea el que disponga de estos conocimientos. Sin embargo, esto sigue implicando un menor grado de exposición a un segundo idioma, aspecto subsanable a partir de la presencia en las aulas de la figura de un especialista en una lengua de signos extranjera o el Sistema Internacional de Signos

\section{References}

Acosta, V.M. (2001). El apoyo a la educación de las personas sordas desde un enfoque bilingüe y bicultural. Innovación Educativa, (11), 221232. https://bit.ly/3ixzTxE

Acosta, V.M. (2003). Un estudio de la sordera como construcción social: visiones externas versus visiones internas. Revista de Logopedia, Foniatría y Audiología, 23(4), 178-194.

https://doi.org/10.1016/S0214-4603(03)75762-3

Alonso, P., Rodríguez, P. \& Echeita, G. (2009). El proceso de un Centro específico de sordos hacia una educación más inclusiva. Colegio Gaudem Madrid. Revista Latinoamericana de Educación Inclusiva, 1(3), 167-187. https://bit.ly/2Zwk3f9

Bellés, R.M. (1995). Presentación: Modelos de atención educativa a los sordos. Infancia y Aprendizaje, 18(69-70), 5-17. https://doi.org/10.1174/021037095321263033

Berent, G.P. (2001). English for deaf students: Assessing and addressing learners' grammar development. International Seminar on Teaching English to Deaf and Hard-of-Hearing Students at Secondary and Tertiary Levels of Education: Proceedings, 124-134.

Blanco, R. \& Duk, C. (2011). Educación inclusiva en América Latina y El caribe. Aula, (17), 37-55. http://dx.doi.org/10.14201/8394

Casanova, M.A. (2011). De la educación especial a la inclusión educativa. Revista Consejo Escolar del Estado. Participación Educativa, (18), 824. https://bit.ly/3mrulam 
Deaño, M. (2004). La Declaración de Salamanca sobre necesidades educativas especiales 10 años después. En G. Echeita y M. A. Verdugo (Coords.), La Declaración de Salamanca Sobre Necesidades Educativas Especiales 10 años después. Valoración y Prospectiva (pp.107-113). Universidad de Salamanca.

De Castro, R.M. (2014). La enseñanza bilingüe en Madrid. Tarbiya, Revista de Investigación e Innovación Educativa, 43(2), 197-209. https://bit.ly/3hyBAJI

Díaz-Estebánez, E. \& Valmaseda, M. (1995). En el camino hacia una educación de calidad para los alumnos y alumnas sordos. Infancia y Aprendizaje, 18(69-70), 45-60. https://doi.org/10.1174/021037095321263060

Domínguez, A.B. (2009). Educación para la inclusión de alumnos sordos. Revista Latinoamericana de Educación Inclusiva, 1(3), 45-61. https://bit.ly/35zeccI

Domínguez, A.B. \& Alonso, P. (2004). La educación de los alumnos sordos hoy: Perspectivas y respuestas educativas. Aljibe.

Domínguez, J. (2016). Una investigación histórica sobre las causas estructurales de la crisis identitaria de la Educación Básica (desde 1945 a 1990). Educatio Siglo XXI, 34(3), 27-46.

https://doi.org/10.6018/j/275751

Echeita, G. \& Verdugo, M.A. (2004). La Declaración de Salamanca Sobre Necesidades Educativas Especiales 10 años después. Valoración y Prospectiva. La Declaración de Salamanca Sobre Necesidades Educativas Especiales 10 años después. Valoración y Prospectiva (pp.57-64). Universidad de Salamanca.

Fernández Agüero, M. (2009). Descripción de los elementos curriculares en la enseñanza bilingüe del inglés: Aproximación a la situación en la Comunidad de Madrid [Tesis Doctoral, Universidad Nacional de Educación a Distancia]. Repositorio Institucional UNED. https://bit.ly/2FC2TFA

Fernández Núñez, L. (2006). ¿Cómo analizar datos cualitativos? Butlletí LaRecerca, 7, 1-13. https://bit.ly/2FuLEqe

Fernández-Viader, M.P \& Fuentes, M. (2004). Education of Deaf Students in Spain: Legal and Educational Politics Developments. Journal of Deaf Studies and Deaf Education, (9)3, 327-332.

https://doi.org/10.1093/deafed/enh035 
Fundación ONCE (2018, 13 de abril). Enseñanza bilingüe (español-inglés) y alumnado con discapacidad: Nuevas barreras y desafíos de inclusión. Discapnet. https://bit.ly/3c94wqR

Garzón, P., Calvo, M.I \& Orgaz, M. (2016). Inclusión educativa. Actitudes y estrategias del profesorado. Revista Española de Discapacidad, 4(2), 25-45. https://doi.org/10.5569/2340- 5104.04.02.02

Giné, C. (2004). La Declaración de Salamanca sobre necesidades educativas especiales 10 años después. Valoración y prospección desde Catalunya. En G. Echeita y M. A. Verdugo (Coords.), La Declaración de Salamanca Sobre Necesidades Educativas Especiales 10 años después. Valoración y Prospectiva (pp.49-55). Universidad de Salamanca.

Gómez Gómez, M.C. (2018). La educación de los sordos de la exclusión a la inclusión. Revista sobre infancia y la adolescencia, (14), 95-106. https://doi.org/10.4995/reinad.2018.7375

González-Gil, F. (2011). Inclusión y atención al alumnado con necesidades educativas especiales. Revista Consejo Escolar del Estado.

Participación Educativa, (18), 60-78. https://bit.ly/35DJe36

Herrera, V. (2014). Alfabetización y bilingüismo en aprendices visuales.

Aportes desde las epistemologías de sordos. Educ. Educ., (17) 1, 135148. https://bit.ly/33pgfO0

Izquierdo, E. (2017). Origen, causas y evolución de la Educación Bilingüe:

el caso de Estados Unidos, Canadá y España [Tesis Doctoral, Universidad Rey Juan Carlos]. Repositorio Institucional URJC. https://bit.ly/3iwaxQu

Julián-de-Vega, C. \& Ávila-López, J. (2018). Políticas lingüísticas europeas y españolas: el camino hacia el cambio en la educación terciaria. Porta Linguarum, Monográfico III, 17-30. https://bit.ly/35C4beR

Kontra, E.H., Csizér, K. \& Piniel, K. (2015). The challenge for Deaf and hard-of-hearing students to learn foreign languages in special needs schools. European Journal of Special Needs Education, 30(2), 141155. https://doi.org/10.1080/08856257.2014.986905

Lankshear, C. \& Knobel, M. (2000). Problemas asociados con la metodología de la investigación cualitativa. Perfiles educativos, 22(87), 6-27. https://bit.ly/35E9874

Laorden Gutiérrez, C. \& Peñafiel Pedrosa, E. (2010). Proyectos bilingües en los centros de la Comunidad de Madrid: Percepción de los equipos 


\section{Garrote Camarena et al. - Aprendizaje L2 en Personas Sordas}

directivos. Revista de Investigación Educativa, 28(2), 325-344. https://bit.ly/3hug31A

Ley 27/2007, de 23 de octubre, por la que se reconocen las lenguas de signos españolas y se regulan los medios de apoyo a la comunicación oral de las personas sordas, con discapacidad auditiva y sordociegas. Boletín Oficial del Estado, 24 de octubre de 2007, núm. 255, pp. 43251 a 43259.

Ley Orgánica 8/2013, de 9 de diciembre, para la mejora de la calidad educativa. Boletín Oficial del Estado, 10 de diciembre de 2013, núm. 295, pp. 97858 a 97921.

Lova, M., Bolarín, M.J. \& Porto, M. (2013). Programas bilingües en Educación Primaria: Valoraciones de docentes. Porta Linguarum, (20), 253-268. https://bit.ly/2FCM7GE

Martínez Miguélez, M. (2006). Validez y confiabilidad en la metodología cualitativa. Paradígma, 27(2), 07-33. https://bit.ly/3hv3W7F

Martín-Pastor, E. \& Durán Martínez, R. (2019). La inclusión educativa en los programas bilingües de educación primaria: Un análisis documental. Revista Complutense de Educación, 30(2), 589-604. https://doi.org/10.5209/RCED.57871

Maxwell, J.A. (2012). Qualitative Research Design. An interactive approach. Sage.

Munarriz, B. (1992). Técnicas y métodos en investigación cualitativa. En E. Abalde \& J.M. Muñoz-Cantero (cords.), Metodología Educativa I. Xornadas de Metodoloxía de Investigación Educativa (pp. 101-116). Universidade de Coruña.

Ohna, S.E. (2003). Education of Deaf Children and the Politics of Recognition. Journal of Deaf Studies and Deaf Education, 8(1), 5-10. https://doi.org/10.1093/deafed/8.1.5

ONU (2006) Asamblea General. Convención sobre los Derechos de las personas con discapacidad: Resolución aprobada por la Asamblea General, 24 de enero 2007, A/RES/61/106. https://bit.ly/3mmpZ3X

Palacios, A. \& Bariffi, F. (2007). La discapacidad como una cuestión de derechos humanos. Una aproximación a la Convención Internacional sobre los Derechos de las Personas con Discapacidad. En E. Trujillo y L.C. Pérez (Dir.) Colección Telefónica Accesible. https://bit.ly/35PK0dJ 
Parrilla Latas, A. (2004). La atención a la diversidad en Andalucía vista desde la Declaración de Salamanca. En G. Echeita y M. A. Verdugo (Coords.), La Declaración de Salamanca Sobre Necesidades Educativas Especiales 10 años después. Valoración y Prospectiva (pp.57-64). Universidad de Salamanca.

Pérez-Llantada, M.C. \& López de la Llave, A. (2001). Metodologías de encuestas: Conceptos básicos y diseños. En S. Fontes, C. GarcíaGallego, A.J. Garriga-Trillo, M.C. Pérez-Llantada y E. Sarriá (Coords.) Diseños de investigación en Psicología (pp. 433-468). Universidad Nacional de Educación a Distancia.

Quay, S. (2005). Education Reforms and English Teaching for the Deaf in Japan. Deafness \& Education International, (7)3, 139-153.

https://doi.org/10.1179/146431505790560338

Real Decreto 126/2014, de 28 de febrero, por el que se establece el currículo básico de la Educación Primaria. Boletín Oficial del Estado, 1 de marzo de 2014, núm. 52, pp. 19349 a 19420.

Real Decreto 1105/2014, de 26 de diciembre, por el que se establece el currículo básico de la Educación Secundaria Obligatoria y del Bachillerato. Boletín Oficial del Estado, 3 de enero de 2014, núm. 3, pp. 169 a 546.

Rodríguez-Ortiz, I.R. (2005). Condiciones para la educación bilingüe de las personas sordas. Revista de Logopedia, Foniatría y Audiología, 25(1), 28-37. https://doi.org/10.1016/S0214-4603(05)75807-1

Sanjosé Gil, A. (2007). El primer tratado de derechos humanos del siglo

XXI: La Convención sobre los derechos de las personas con discapacidad. Revista Electrónica de Estudios Internacionales, (13), 1-26. https://bit.ly/33s2JJr

Soriano de Gracia, V. (2011). La educación inclusiva en Europa. Revista Consejo Escolar del Estado. Participación Educativa, (18), 35-45. https://bit.ly/2ZDefR0

Sotoca, E. (2014). La repercusión del bilingüismo en el rendimiento académico en alumnos de colegios públicos de la Comunidad de Madrid. Revista Complutense de Educación, 25(2), 481-500. https://doi.org/10.5209/rev_RCED.2014.v25.n2.41732

Taylor, S.J. \& Bogdan, R. (1987). La entrevista en profundidad. En Introducción a los métodos cualitativos en investigación. La búsqueda de los significados (pp. 100-132). Paidos. 


\section{Garrote Camarena et al. - Aprendizaje L2 en Personas Sordas}

UNESCO (1994). Declaración de Salamanca y Marco de Acción para las Necesidades Educativas Especiales. Aprobado por la Conferencia Mundial Sobre Necesidades Educativas Especiales: Acceso y Calidad. Salamanca, 7-10 de junio de 1994: UNESCO

Verdugo, M.A. (2011). Implicaciones de la Convención de la ONU (2006) en la educación de los alumnos con discapacidad. Revista Consejo Escolar del Estado. Participación Educativa, (18), 25-34. https://bit.ly/32wx9L1

Inmaculada Garrote Camarena is $\mathrm{PhD}$ and visiting lecturer in the Department of Education, Language, Culture and Arts, Historical-legal and Humanistic Sciences and Modern Languages at Faculty of Social and Legal Sciences at Rey Juan Carlos University, Spain.

Ricardo Moreno Rodríguez is Associate professor in the Department of Education, Language, Culture and Arts, Historical-legal and Humanistic Sciences and Modern Languages at Faculty of Social and Legal Sciences at Rey Juan Carlos University, Spain.

Francisco Reyes Téllez is Senior Lecturer in the Department of Education, Language, Culture and Arts, Historical-legal and Humanistic Sciences and Modern Languages at Faculty of Social and Legal Sciences at Rey Juan Carlos University, Spain.

Contact Address: Inmaculada Garrote Camarena, Rey Juan Carlos University, Faculty of Social and Legal Sciences, Paseo de los Artilleros, 0, 28032 Madrid, Spain. Email: inmaculada.garrote@urjc.es 